\title{
BDNF Levels Significantly Lower in Mothers of Autistic Individuals
}

\author{
AJ Russo, ${ }^{1 *}$ Albert Mensah, ${ }^{2}$ Judith Bowman, ${ }^{2}$ \\ ${ }^{1}$ Drew University, USA \\ ${ }^{2}$ Mensah Research Institute, USA
}

\begin{abstract}
Brain Derived Neurotrophic Factor (BDNF) is a growth factor linked depression, schizophrenia and Alzheimer's disease. We used immune arrays to quantitate levels of BDNF in the plasma of mothers of autistic children and neurotypical controls. We found that BDNF levels significantly lower in mothers of autistic individuals compared to controls. This data suggests that mothers of individuals with autism have decreased BDNF levels which may be associated with the etiology of autism in their children.
\end{abstract}

Keywords: ASD, BDNF, Plasma, Psychiatric disorders

\section{Introduction}

Autism Spectrum Disorders (ASD) are characterized by symptoms of dysfunctional social interactivity, communication, and many stereotyped behaviors ${ }^{1}$ and diagnosis is based solely on behavioral criteria. Brain Derived Neurotrophic Factor (BDNF) is a member of the neurotrophin family of growth factors, which are related to the canonical nerve growth factor. Neurotrophic factors are found in the brain and the periphery. Various studies have shown possible links between BDNF and conditions, such as depression ${ }^{2}$ schizophrenia ${ }^{3}$ Alzheimer's disease ${ }^{4}$ Huntington's disease ${ }^{5,6}$ Rett syndrome $^{7}$ and dementia. ${ }^{8}$ Abnormal expression of BDNF is associated with neurologic and psychiatric disorders, including Fragile $\mathrm{X}$ syndrome, epilepsy, Parkinson's disease, Alzheimer's disease, schizophrenia and depression. ${ }^{9-11}$ We used immune-arrays to quantitate levels of BDNF in the plasma of mothers of autistic children and neurotypical controls.

\section{Methods}

\section{Subjects}

Plasma BDNF was measured in 15 mothers of autistic children and 12 age and gender similar neurotypical controls. Subject plasmas were obtained from the Autism Genetic Resource Exchange (AGRE)**. This study was approved by the IRB of the Health Research Institute.

\section{Plasma}

All plasma was received frozen and immediately placed at -70C before Immunoassay analysis.

\section{Immuno-array assays}

Immuno-array assays, as previously described, ${ }^{12}$ were performed by Ray Biotech, Inc, Peachtree Corners, GA. 30092.

\begin{tabular}{|l|l|}
\hline \hline Quick Response Code: & *Corresponding author: AJ Russo, Visiting Assistant Professor of Biology, Drew University, \\
Madison, NJ 07940, Research Director, Mensah Research Institute, Warrenville, Il 60555, USA \\
Received: 27 September, 2021 \\
Citation: Russo AJ, Mensah A, Bowman J. BDNF Levels Significantly Lower in Mothers of Autis- \\
tic Individuals. SOJ Pedia Clin Neonato. 2021;1(3):1-2. DOI: \\
$10.53902 / S O J P C N .2021 .01 .000512$
\end{tabular}




\section{Statistics}

Statistical analysis was done using T-tests with $95 \%$ confidence levels.

**We gratefully acknowledge that all autism family serums were provided by the Autism Genetic Resource Exchange (AGRE) Consortium and the participating AGRE families. The Autism Genetic Resource Exchange is a program of Cure Autism Now and is supported, in part, by grant MH64547 from the National Institute of Mental Health to Daniel H Geschwind (PI)

\section{Results}

We found that BDNF levels significantly lower in mothers of autistic individuals compared to controls Figure 1.

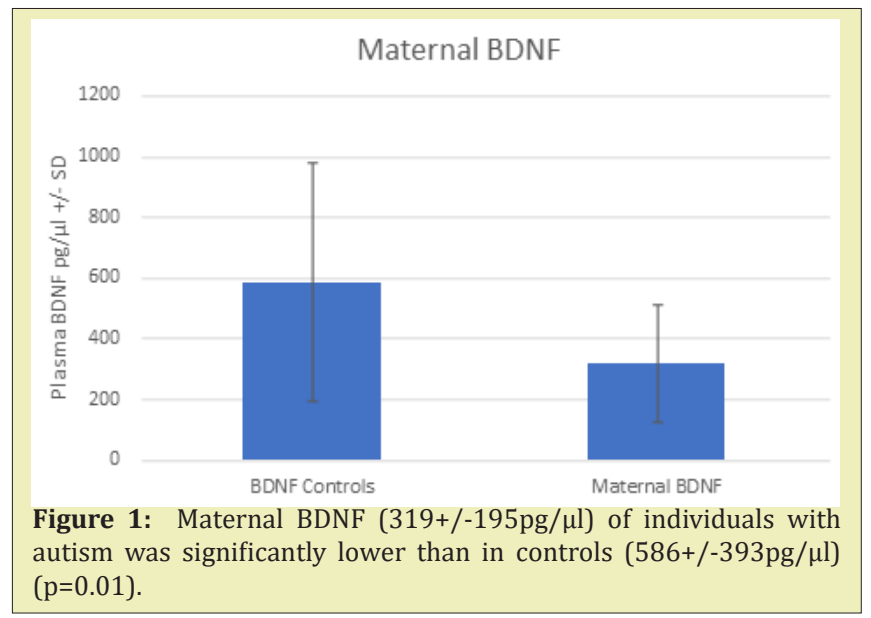

\section{Discussion}

A meta-analysis of blood BDNF in 887 patients with ASD and 901 control subjects demonstrated significantly higher BDNF levels in ASD compared to controls, ${ }^{12}$ and another meta-analysis included 19 studies with 2896 unique participants. Random-effects meta-analysis of all 19 studies showed that children with ASD had significantly increased peripheral blood levels of BDNF compared with healthy controls. ${ }^{13}$ These observed relationships are most likely the result of impact of pathogenesis rather than inheritance because elevated BDNF was not associated with BDNF genepolymorphism. ${ }^{14}$ Our results indicate that mothers of autistic individuals have decreased BDNF and suggests a metabolic over-compensation effect leading to her autistic child's higher BDNF production.

\section{Conclusion}

This data suggests that mothers of individuals with autism have decreased BDNF levels. This may be associated with the etiology of autism in their children.

\section{Acknowledgments}

None.

\section{Funding}

None.

\section{Conflicts of Interest}

Author declares that there is no conflict of interest.

\section{References}

1. Lord C, Cook EH, Leventhal BL, et al. Autism spectrum disorders. Neuron. 2000;28(2):355-363.

2. Dwivedi Y. Brain-derived neurotrophic factor: role in depression and suicide. Neuropsychiatric Disease and Treatment. 2009;5:433-449.

3. Xiu MH, Hui L, Dang YF, et al. Decreased serum BDNF levels in chronic institutionalized schizophrenia on long-term treatment with typical and atypical antipsychotics. Progress in Neuro-Psychopharmacology \& Biological Psychiatry. 2009;33(8):1508-1512.

4. Maina G, Rosso G, Zanardini R, et al. Serum levels of brain-derived neurotrophic factor in drug-naïve obsessive-compulsive patients: a case-control study. Journal of Affective Disorders. 2010;122(1-2):174178.

5. Zuccato C, Cattaneo E. Brain-derived neurotrophic factor in neurodegenerative diseases. Nature Reviews Neurology. 2009;5(6): 311-322.

6. Zajac MS, Pang TY, Wong N, et al. Wheel running and environmental enrichment differentially modify exon-specific BDNF expression in the hippocampus of wild-type and pre-motor symptomatic male and female Huntington>s disease mice. Hippocampus. 2010;20(5):621636.

7. Zeev BB, Bebbington A, Ho G, et al. The common BDNF polymorphism may be a modifier of disease severity in Rett syndrome. Neurology. 2009;72(14):1242-1247.

8. Arancio 0, Chao MV. Neurotrophins, synaptic plasticity and dementia. Current Opinion in Neurobiology. 2007;17(3):325-330.

9. Pezet $\mathrm{S}$, Malcangio M. Brain-derived neurotrophic factor as a drug target for CNS disorders. Expert OpinTher Targets. 2004;8:391-399.

10. Binder DK, Scharfman HE. Brain-derived neurotrophic factor. Growth Factors. 2004;22(3):123-131.

11. Hashimoto K, Shimizu E, Iyo M. Critical role of brain-derived neurotrophic factor in mood disorders. Brain Res Brain Res Rev. 2004;45:104-114.

12. Saghazadeh A, Rezaei N. Brain-Derived Neurotrophic Factor Levels in Autism: A Systematic Review and Meta-Analysis. J Autism Dev Disord. 2017;47(4):1018-1029.

13. Qin XY, Feng JC, Cao C, et al. Association of Peripheral Blood Levels of Brain-Derived Neurotrophic Factor with Autism Spectrum Disorder in Children: A Systematic Review and Meta-analysis. JAMA Pediatr. 2016;170(11):1079-1086.

14. Meng WD, Sun SJ, Yang J, et al. Elevated Serum Brain-Derived Neurotrophic Factor (BDNF) but not BDNF Gene Val66Met Polymorphism Is Associated with Autism Spectrum Disorders. Mol Neurobiol. 2017;54(2):1167-1172. 